\title{
Erratum: Coherent forward broadening in cold atom clouds [Phys. Rev. A 93, 023407 (2016)]
}

R. T. Sutherland and F. Robicheaux

(Received 20 September 2016; published 30 September 2016)

DOI: 10.1103/PhysRevA.94.039904

There is a typo in the second term of Eq. (14). It should read

$$
N+N(N-1)\left(\frac{1}{2 \pi \sigma^{2}}\right)^{3} \iint d^{3} x_{1} d^{3} x_{2} \exp \left(\frac{-1}{2 \sigma^{2}}\left\{\xi\left(x_{1}^{2}+x_{2}^{2}+y_{1}^{2}+y_{2}^{2}\right)+\frac{z_{1}^{2}+z_{2}^{2}}{\xi^{2}}\right\}\right) \exp \left(i\left\{\boldsymbol{k}-\boldsymbol{k}_{0}\right\} \cdot\left\{\boldsymbol{r}_{1}-\boldsymbol{r}_{2}\right\}\right) \text {. }
$$

This is a typographical error, and does not effect any other part of the paper. 